\title{
Impaired Statistical Learning with \\ Mathematical Learning Difficulties
}

\author{
Sharon Levy ${ }^{1}$, Nicholas B. Turk-Browne, ${ }^{2}$ Liat Goldfarb ${ }^{1}$ \\ ${ }^{1}$ Edmond J. Safra Brain Research Center for the Study of Learning Disabilities, \\ University of Haifa \\ 2 Department of Psychology, Yale University
}

Correspondence concerning this article should be addressed to:

Dr. Liat Goldfarb

E.J.S Brain Research Center for the study of learning disabilities University of Haifa, Mount Carmel, Haifa, Israel 31905

Email: goldfarb@edu.haifa.ac.il

Keywords: numerical cognition; statistical learning; mathematical learning difficulties 


\section{ABSTRACT}

Rapid extraction of temporal and spatial patterns from repeated experience is known as statistical learning (SL). Studies on SL show that after few minutes of exposure, observers exhibit knowledge of regularities hidden in a sequence or array of objects. Previous findings suggest that SL might relate to numerical processing mechanisms. Hence, the current study examines for the first time SL in a population with a deficiency in the numerical system: individuals with mathematical learning difficulties (MLD). The results revealed that $S L$ was significantly worse in the MLD group than in a control group. In addition, whereas the control group showed reliable SL above chance, the MLD group did not. Because learned regularities can broadly facilitate cognitive processing, individuals with MLD may thus suffer from additional behavioral challenges beyond their numerical difficulties. 


\section{INTRODUCTION}

Humans have lived in very similar natural environments throughout evolutionary history. Because of this stability, sensory systems in the brain have adapted to the kinds of input that are most likely to appear (statistical regularities). For example, the receptive field properties of the primary visual cortex can be regenerated from scratch by decomposing a set of natural images into sparse independent components (Olshausen \& Field, 1996). Analogously, we repeatedly encounter particular natural and artificial environments during our lifetime, and each of these environments has its own idiosyncratic regularities. For example, when moving to a new town, the sequence of landmarks encountered when navigating to the office is likely different than before. Although extensive experience is required for some types of perceptual learning (Gilbert, Sigman, \& Crist, 2001; Watanabe, Nanez, \& Sasaki, 2001), temporal sequences and spatial configurations of objects in our environments can be learned surprisingly quickly.

This rapid extraction of temporal and spatial patterns from repeated experience is known as statistical learning (SL; see Aslin \& Newport, 2012; Frost, Armstrong, Siegelman, \& Christiansen, 2015). In studies of SL, observers are presented with a sequence or array of objects that, unbeknownst to them, contains hidden regularities in which objects appear nearby in time or space, respectively. Critically, the only clue that objects are related comes from their statistical co-occurrence over repeated experiences. In other words, at any time in the sequence or location in the array, an object could appear next to several other objects; but across extended sequences and multiple arrays, it is more likely to appear with one or more specific others. After a few minutes of exposure, observers exhibit knowledge of these regularities in familiarity, response time, and brain imaging measures. 
Research suggests that SL is ubiquitous: it operates in multiple modalities and dimensions (Conway \& Christiansen, 2006; Turk-Browne \& Scholl, 2009); over many features such as shape (e.g., Fiser \& Aslin, 2001), spatial location (e.g., Chun \& Jiang, 1998), color (e.g., Turk-Browne, Isola, Scholl, \& Treat, 2008), and action (e.g., Baldwin, Andersson, Saffran, \& Meyer, 2008); throughout development from infants (Kirkham, Slemmer, \& Johnson, 2002) to the elderly (Campbell, Zimerman, Healey, Lee, \& Hasher, 2012); and in non-human species (e.g., Toro \& Trobalón, 2005). In addition, SL is more robust for attended input but proceeds automatically, without intent or conscious awareness of regularities (Turk-Browne, Jungé, \& Scholl, 2005; Emberson, Conway, \& Christiansen, 2011; Campbell et al., 2012; Musz, Weber, \& Thompson-Schill, 2015). For example, during working memory or other tasks, observers incidentally learn regularities that are irrelevant to the task, and report no awareness or knowledge of the regularities (Turk-Browne et al., 2005; Turk-Browne, Scholl, Chun, \& Johnson, 2009).

$\mathrm{SL}$ is related to other cognitive processes. For example, SL interacts with statistical summary perception (SSP) — our ability to immediately perceive summary properties (e.g., average size) from a set of objects (Ariely, 2001; Chong \& Treisman, 2005; Alvarez \& Oliva, 2008). SL and SSP have been shown to mutually interfere with each other: estimating the mean orientation of a set of lines (a form of SSP) impairs learning of regularities in which orientations tend to appear adjacent in space, and these mean estimates improve when learning is hindered by removing the orientation regularities (Zhao, Ngo, McKendrick, \& Turk-Browne, 2011). These findings suggest that SL and SSP rely upon similar statistical computations, which cannot be used simultaneously by both processes. 
Beyond summary statistics about the features of objects, SL also interacts with the extraction of other set properties, such as numerosity. Estimates of the number of colored circles in an array are reliably worse when across trials the arrays contain repeated pairs of colors (allowing for SL) compared to when no color regularities are present (impeding $S L)$; in the reverse direction, numerosity estimation relative to control tasks impairs $S L$ of color pairs (Zhao \& Yu, 2016). Thus, as with SL and SSP, SL and numerosity estimation interfere with each other, suggesting that they may also rely upon a shared mechanism. Neuroimaging findings are consistent with this possibility. Namely, the intraparietal sulcus (IPS) is specialized for processing numbers and quantities (e.g., Dehaene, Piazza, Pinel, \& Cohen, 2003; Piazza, Izard, Pinel, Le Bihan, \& Dehaene, 2004; Piazza, Pinel, Le Bihan, \& Dehaene, 2007) and adjacent Brodmann area 40 is more strongly activated by streams of shapes with vs. without regularities (Turk-Browne et al., 2009). Moreover, IPS activity in response to an object that predicts what will come next in a sequence as a result of SL correlates with the magnitude of behavioral priming for the next object (Turk-Browne, Scholl, Johnson, \& Chun, 2010).

In sum, both behavioral and neuroimaging findings point to a relationship between SL and numerical processing. This raises questions about how SL would be affected by impairments in numerical processing associated with mathematical learning difficulties (MLD). Individuals with MLD have mathematical skills far below what would be predicted based on their age, intelligence, and education (e.g., Butterworth, Varma, \& Laurillard, 2011). They can have difficulties in simple counting tasks (Geary et al., 2009), deficiencies in processing numerical quantities and magnitudes, failures in number identification, and have trouble associating magnitudes with symbols (see Rubinsten \& Henik, 2009). The 
estimated prevalence of MLD is about $3-6 \%$ of school-age children (similar to the prevalence of dyslexia and ADHD), and in many cases the numerical difficulties persist into adulthood (e.g., Shalev, Auerbach, Manor \& Gross-Tsur, 2000; Shalev, Manor \& GrossTsur, 2005). Difficulties in the arithmetic domain affect the numerical performance of the individual at school and in everyday life (Butterworth, 2009). Simple tasks, such as calculating change in a grocery store, can be difficult and confusing for people with MLD even in adulthood. MLD has been characterized extensively in prior work (e.g., Price, \& Ansari, 2013), including evidence that dysfunction of IPS may be partly responsible (Cohen-Kadosh et al., 2007; Price, Holloway, Räsänen, Vesterinen, \& Ansari, 2007).

The goal of the current study is to examine SL in a population with MLD. Our hypothesis is that because numerical processes and SL might rely on the same underlying computations, and MLD results from a deficit in these computations, individuals with MLD might show weaker SL than a matched control group with no learning difficulties.

\section{METHOD}

\section{Participants}

Sixteen females with MLD aged 22-33 (M=26.25, SD=3.51) and a matched control group of 16 female adults with no learning difficulties or attention disorders aged 20-35 $(M=25.19, S D=4.04)$, participated in the experiment. There was no significant difference in age between the two groups $[t(30)=.79, p=.43]$. This sample size per group is comparable to prior SL studies (Turk-Browne et al., 2008; Zhao et al., 2011). All participants provided written informed consent and were paid for their participation (approximately $\$ 16-22$ ). The Ethical Committee of Haifa University approved all the study procedures. 


\section{Classification and assessment}

Classification of the MLD group was performed using computerized numerical tests from the "Israeli learning function diagnosis system" (MATAL, 2007) for high-school and higher-education students ${ }^{1}$. In addition, participants in the MLD group completed a short interview with a learning disability diagnostician, in which they described significant difficulties in the arithmetic field, from the early years of elementary school until the current time.

All participants in the study underwent a series of tests to assess abilities including: reading using the one-minute test for words and pseudo-words, (Shatil, 1997a, 1997b), verbal retrieval using the phonological and semantic fluency test (Kave 2006), non-verbal reasoning using the Raven Progressive Matrices (Raven, 1960), basic arithmetic abilities using the 2-minute calculation test (Openhaim-Biton \& Breznitz, 2004), and attention using the attention questionnaire based on the ADHD Diagnostic and Statistical Manual of Mental Disorders (DSM-V) criteria. The order of the tests was counterbalanced except of the attention questionnaire that appeared last (to avoid a priming effect).

\footnotetext{
${ }^{1}$ This system was developed by the National Institute for Testing and Evaluation, and contains a large set of nationally normalized tests that is used to diagnose learning difficulties, including MLD. Participants from the MLD group underwent two numerical tests: simple calculation tasks (e.g., $2+2=4,2{ }^{\star} 2=5,8-4=4,6: 4=2$ ) and procedural knowledge calculation tasks (e.g., $750+10=760,204-5=201,20 * 20=400,400: 5=45$ ). In both tasks the participants were asked to report whether the equation appearing on the screen was correct. Each task produced two measures: reaction time and accuracy. To be included in the MLD group, the average of all four measures had to be below the $20^{\text {th }}$ percentile (no individual measure was above the $33^{\text {rd }}$ percentile).
} 
A

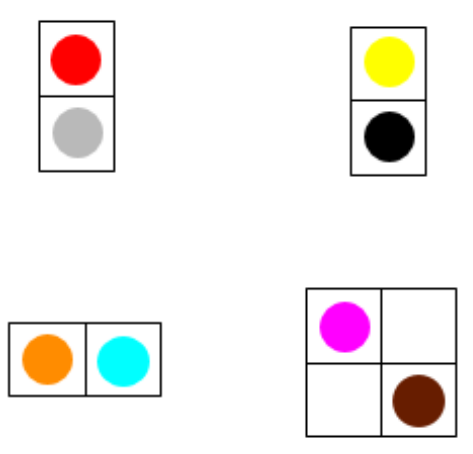

Examples of pairs of colored circles

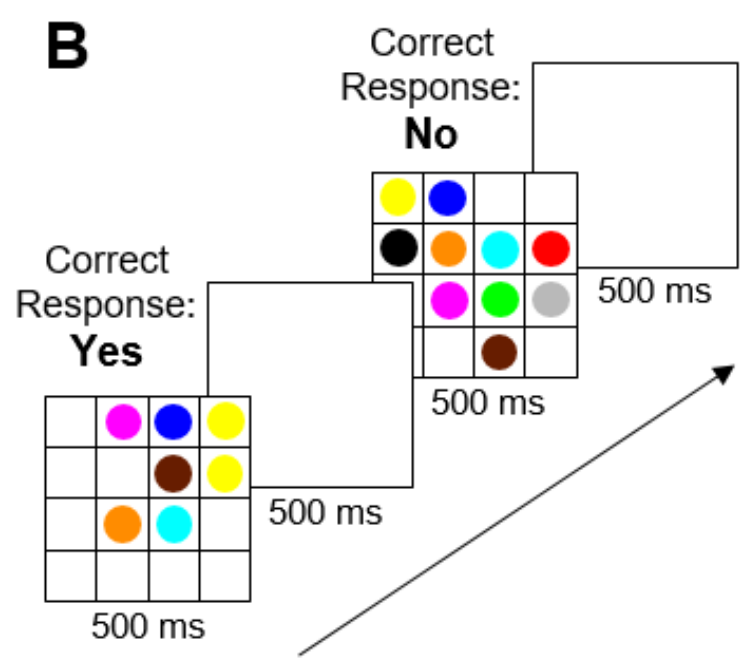

Exposure phase - duplicate task: Are there two circles of identical color in the display?

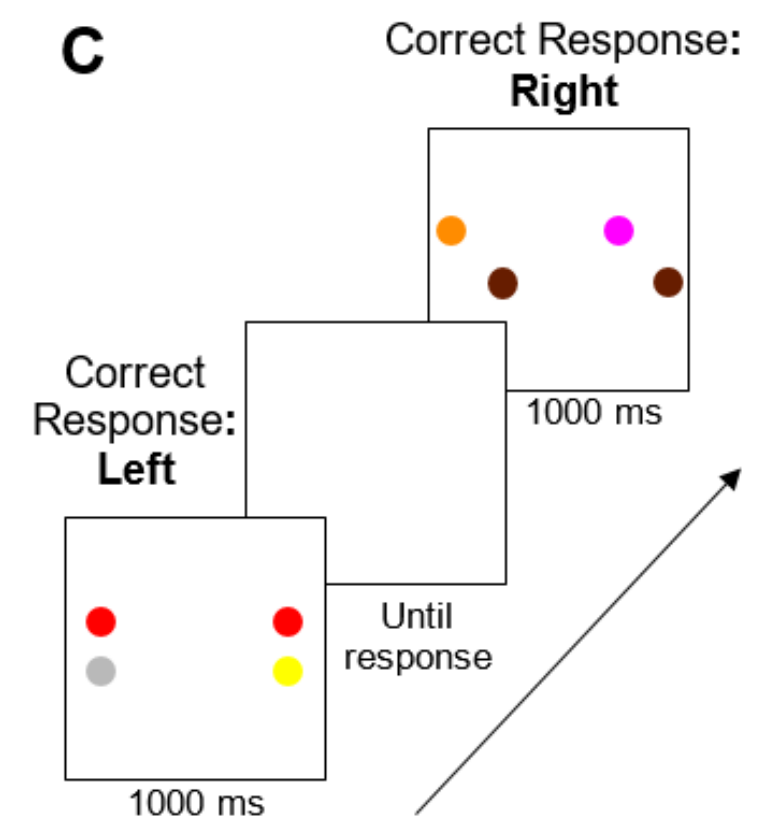

Test phase: Which pair is more familiar?

Figure 1: (A) Examples of the colored pairs. (B) An example of trial sequence of the exposure phase and the corresponding correct responses. Participants were asked to report whether there were two circles of identical color in the display. (C) An example of trial sequence of the test phase and the corresponding correct responses. Participants were asked to report which of two pairs (correct and foil) was more familiar. 


\section{Stimuli}

Circular disks subtending $1.4^{\circ}$ of visual angle appeared on a white screen (255/255/255) in one of ten colors (color name and R/G/B values: red, 255/0/0; green, 0/255/0; blue, 0/0/255; yellow, 255/255/0; magenta, 255/0/255; cyan, 0/255/255; gray, 185/185/185; orange, 255/140/0; brown, 103/29/0; black, 0/0/0). Eight circles were randomly selected for each participant to create four pairs, which were grouped together into a fixed horizontal, vertical, and diagonal structure (Figure 1A).

In every trial, a display of 2 to 10 (excluding 6 ) colored circles appeared on the screen. The display was created on an invisible $4 \mathrm{X} 4$ grid (subtending $18.1 \times 18.1^{\circ}$ ) . Displays with odd numbers of circles $(3,5,7,9)$ contained unique pairs $(1,2,3,4$ pairs, respectively) and one single circle. Half of the displays with even numbers of circles $(4,8)$ contained only pairs $(2,4$, respectively), and the other half contained both pairs $(1,3$, respectively) and two single circles. Displays with two circles always contained two single circles to avoid a color pair being presented by itself. Displays with 10 circles contained the four unique pairs and the two single circles. Each number of circles appeared 50 times, for a total of 400 trials, presented in a random order for each participant. In $20 \%$ of the displays, a randomly selected pair was modified so both circles appeared in the same color.

\section{Apparatus}

An HP Compaq computer with a 22-inch Samsung monitor was used to present stimuli and to collect the data. The experiment was presented using Matlab (Mathworks, Natick, MA) and the Psychophysics Toolbox (Brainard, 1997; Pelli, 1997). The participants sat at a distance of about $60 \mathrm{~cm}$ from the screen and a keyboard was placed next to it. 


\section{Procedure}

In the exposure phase, displays of colored circles appeared for $500 \mathrm{~ms}$, followed by a blank interval of $500 \mathrm{~ms}$. The participants pressed as accurately as possible the "/" button if two circles had the same color and the "z" if not (Figure 1B). In the test phase two pairs of circles appeared randomly for $1000 \mathrm{~ms}$ (Figure 1C). Participants were asked to decide which side contained two colors whose combination was more familiar based on the first part of the experiment. They pressed either the "1" or "0" button on top of the keyboard for the left or right side. Two foils were created for each of the four exposure pairs, one for each of the two colors. Each pair was tested twice separately against both foils, for a total of 16 test trials. Each participant was tested individually for about 20 minutes in total.

\section{RESULTS}

\section{Cognitive assessment}

As shown in Table 1, independent samples t-tests revealed no significant difference between the MLD group and the control group in any assessment ( $p s>0.15)$ except for the 2-minute calculation test. In this test, as expected, the MLD group performed significantly worse compared to the control group. 


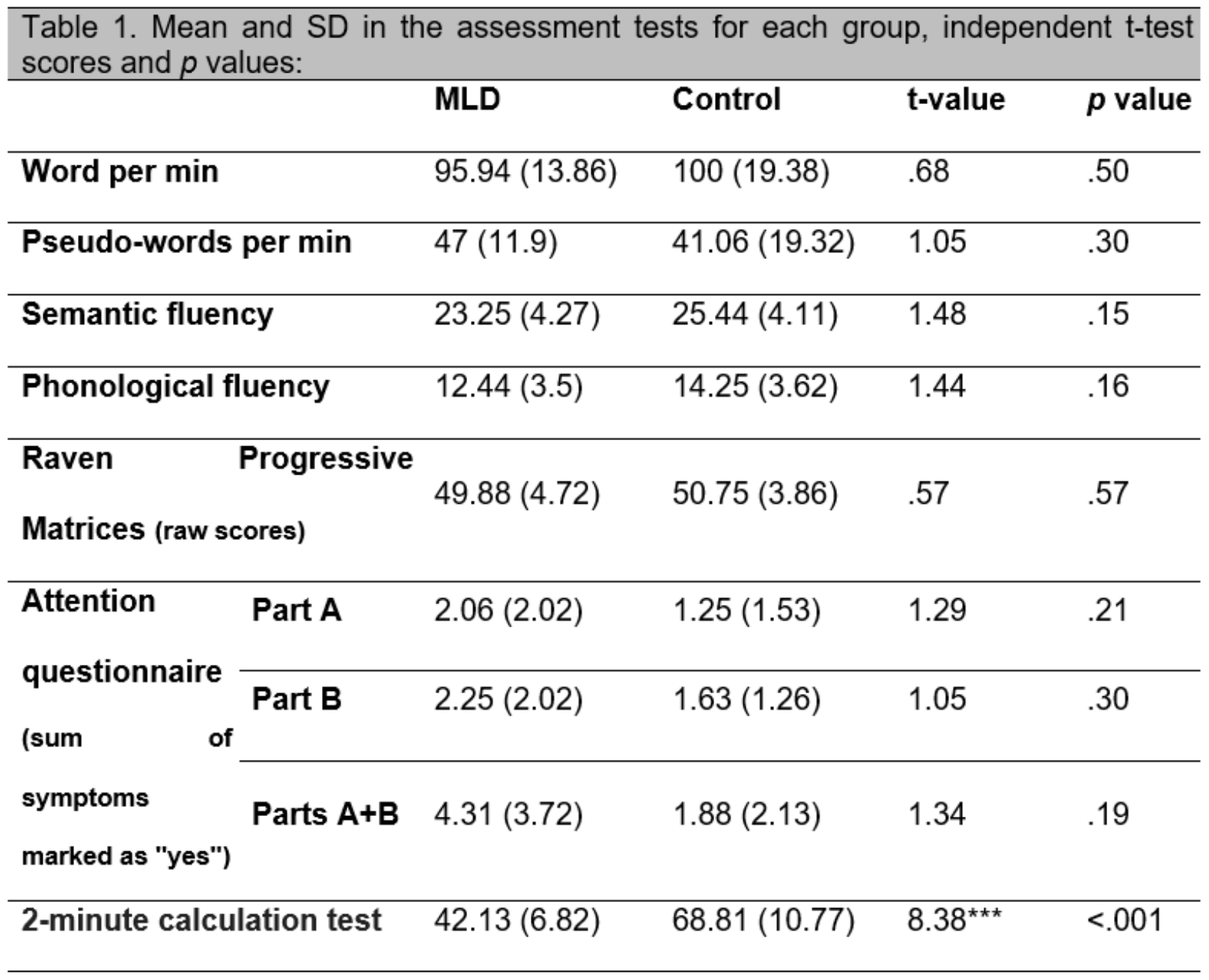

\section{Color duplication detection}

All participants performed above $90 \%$ accuracy in detecting color duplicates during the exposure phase. In addition, $A^{\prime}$ was calculated for each participant as a non-parametric measure of sensitivity (given the high accuracy), treating a "yes" response to duplicate trials as a hit and a "yes" response to non-duplicate trials as a false alarm. No significant difference was found between the MLD group $(M=0.98, S D=0.01)$ and the control group $(\mathrm{M}=0.97, \mathrm{SD}=0.01)[t(30)=0.91, p=0.37$, Cohen's $d=0.32]$. 


\section{Familiarity test}

An independent samples t-test was conducted for accuracy in choosing the pair over the foil across trials in the test phase. As shown in Figure 2, SL was significantly worse in the MLD group $(M=52.73, S D=14.43)$ than in the control group $(M=63.28, S D=13.86)$ $[t(30)=2.11, p<.05$, Cohen's $d=0.75]$. In addition, one-sample t-tests against chance $(50 \%)$ revealed evidence of learning in the control group $\left[t(15)=3.83, p<.001, d^{\prime \prime}=0.96\right]$ but not in the MLD group $\left[t(15)=0.76, p=.46, d^{\prime \prime}=0.19\right]$.

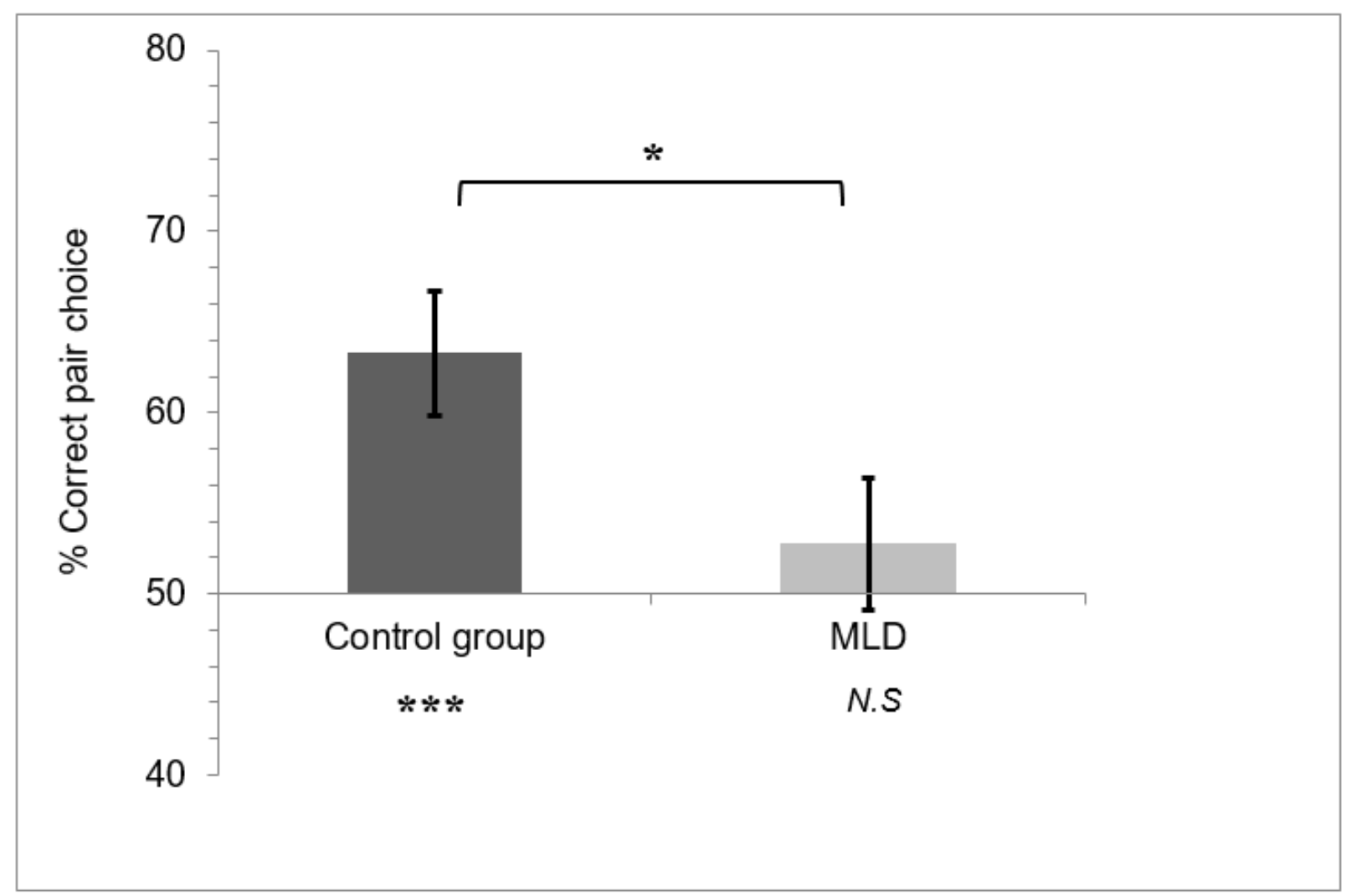

Figure 2. Statistical learning for MLD \& Control groups. Error bars represent \pm one standard error of the mean; ${ }^{\star} p<.05,{ }^{\star \star \star} p<.001, N . S=$ not significant. 


\section{Bayesian analysis}

The lack of reliable test performance in the MLD group could be due to impaired SL in this population or data insensitivity (e.g., noise). To distinguish these two possibilities, Bayesian statistical analyses were conducted. Given the relatively small number of participants, these analyses provide further context and support for the results.

Bayes factors (BF) were calculated using JASP statistical software (version 0.9.2 with default priors; Rouder, Morey, Speckman, \& Province, 2012). The analysis suggested moderate evidence for the difference in $S L$ between the two groups, $B F_{10}=3.346$. In addition, very strong support was found for the research hypothesis that the control group would show SL above chance (50\%), $\mathrm{BF}_{10}=50.288$, and moderate evidence that $\mathrm{SL}$ in the MLD group was not above chance (50\%), $\mathrm{BF}_{10}=0.328$.

\section{DISCUSSION}

The current study examined a new aspect of the relationship between SL and arithmetic processing, while addressing a population with a numerical deficit in the form of mathematical learning difficulties. This population has severe difficulties in understanding and processing numbers and quantities (e.g., Butterworth, 2009).

A group of adults with MLD and a matched control group performed a visual SL task. Both orthodox and Bayesian statistical analyses showed SL above chance for participants with no learning difficulties. This is consistent with previous studies that demonstrated SL in a variety of tasks (e.g., Chun \& Jiang, 1998; Fiser \& Aslin, 2001; Conway \& Christiansen, 2006; Turk-Browne et al., 2008). More importantly, both orthodox and Bayesian statistical analyses also suggested impaired SL in the population with MLD 
relative to individuals without such difficulties, and furthermore indicated that no SL occurred in MLD relative to chance.

Our findings are in line with previous studies that reported a connection between SL and tasks including SSP (Zhao et al., 2011) and numerosity estimation (Zhao \& Yu, 2016). The present findings and these previous studies together suggest that this connection may result from reliance on a common underlying mechanism. Namely, the same processes that help keep track of discrete and/or continuous quantities may also be required to track and update probabilities over time, but not both at the same time. In the MLD population, this mechanism may be impaired, explaining their numerical difficulties, but also having the co-morbid symptom of reducing or eliminating SL.

Another explanation for the connection between SL and numerical processing might be related to other characteristics shared by these two abilities. SL (unknowingly) requires differentiating each item in an array and learning the relations between individual items (Turk-Browne et al., 2009). This process of differentiating and assessing relations is also relevant while dealing with numbers and quantities, for example, when comparing two quantities and deciding which is larger. In addition, repetitive exposure to numbers and experience with numerical manipulations contribute to a more efficient numerical processing (Siegler \& Opfer, 2003). Therefore, learning of regularities from repeated experience in SL might support the process of numerical development.

From an evolutionary point of view, it has been suggested that the arithmetic system is an evolved mechanism that has ancestral origins in other brain mechanisms (Anderson, 2010). The core hypothesis of mental reuse in this work is "that evolutionary considerations might often favor reusing existing components for new tasks over developing new circuits 
de novo" (p. 246). It has been suggested that the numerical system might reuse other mechanisms, such as the finger representation mechanism or the size evaluation mechanism (e.g., Cantlon, Platt, \& Brannon, 2009; Aisenberg \& Henik, 2010; Anderson, 2010). Hence, it is possible that the processes underlying the numerical system and SL abilities are at least partly shared.

With regard to individuals with MLD, the present findings provide another indication that they suffer not only from mathematical difficulties (e.g., Butterworth, 1999; Geary et al., 2009) but also from a more general deficiency. For example, individuals with MLD have poor visuo-spatial abilities, such as in physical and mental number line bisection tasks (Ashkenazi \& Henik, 2010). Moreover, individuals with MLD have difficulties with mental rotation (Szucs, Devine, Soltesz, Nobes, \& Gabriel, 2013; Skagerlund \& Träff, 2014) and with visual working memory (Rotzer et al., 2009; Szucs et al., 2013).

The visual SL task we used involved spatial regularities in color arrays, and thus the observed deficit may be another case of visuo-spatial difficulties in MLD. This can be tested in future studies by employing different kinds of SL tasks that lack a visuo-spatial component, such as temporal SL in visual (Fiser \& Aslin, 2002) and auditory (Saffran, Aslin, \& Newport, 1996) modalities. These future directions would help characterize whether MLD results in a general impairment in SL, or whether the deficit is specific to the spatial dimension and visual modality. In addition, the links between SL and other cognitive processes, such as reading (Frost, Siegelman, Narkiss, \& Afek, 2013; Spencer, Kaschak, Jones, \& Lonigan, 2015), may point to other potential deficits in MLD.

Indeed, the current study suggests that individuals with MLD might have broad differences in cognition. Learned regularities can facilitate cognitive processing, for 
example, leading to increased working memory capacity (Brady, Konkle, \& Alvarez, 2009), faster visual search (Chun \& Jiang, 1998), and improved object recognition (Bays, TurkBrowne, \& Seitz, 2015). This facilitation could impact real-word visuo-spatial behavior. When entering a new workplace, we typically know where to reach for the light switches or where coffee mugs and silverware can be found in the kitchen. When in a new supermarket, we can shop efficiently knowing the plausible location of what we need to buy in relation to other products. Thus, beyond central numerical difficulties, impaired SL in individuals with MLD may create new challenges in everyday life. 


\section{Funding}

This research was supported by fellowships from the Azrieli Foundation (S.L.) and by Grant 2011315 from the U.S-Israel Binational Science Foundation (BSF) (L.G. \& N.T.B).

\section{Declaration of Conflicting Interests}

The authors declared that they had no conflicts of interest with respect to their authorship or the publication of this article. 


\section{REFERENCES}

Aisenberg, A., \& Henik, A. (2010). Re-use or re-function? Behavioral and Brain

Sciences, 33, 266-267.

Alvarez, G. A., \& Oliva, A. (2008). The representation of simple ensemble visual features outside the focus of attention. Psychological Science, 19, 392-398.

American Psychiatric Association. (2013). Diagnostic and Statistical Manual of Mental Disorders (5th ed.). Arlington, VA: American Psychiatric Publishing.

Anderson, M.L (2010). Neural reuse: A fundamental organizational principle of the brain. Behavioral and Brain Sciences, 33, 245-313.

Ariely, D. (2001). Seeing sets: Representation by statistical properties. Psychological Science, 12, 157-162.

Ashkenazi, S., \& Henik, A. (2010). A disassociation between physical and mental number bisection in developmental dyscalculia. Neuropsychologia, 48, s28612868.

Aslin, R. N., \& Newport, E. L. (2012). Statistical learning: From acquiring specific items to forming general rules. Current Directions in Psychological Science, 21, 170176.

Baldwin, D., Andersson, A., Saffran, J., \& Meyer, M. (2008). Segmenting dynamic human action via statistical structure. Cognition, 106, 1382-1407.

Bays, B. C., Turk-Browne, N. B., \& Seitz, A. R. (2015). Dissociable behavioural outcomes of visual statistical learning. Visual Cognition, 23, 1072-1097. 
Brady, T. F., Konkle, T., \& Alvarez, G. A. (2009). Compression in visual working memory: Using statistical regularities to form more efficient memory representations. Journal of Experimental Psychology: General, 138, 487-502.

Brainard, D. H. (1997). The psychophysics toolbox. Spatial Vision, 10, 433-436.

Butterworth, B. (1999). What Counts: How Every Brain is Hardwired for Math. New York: Free Press.

Butterworth, B. (2009). Developmental dyscalculia. In J. Reed \& J. Warner-Rogers (Eds.), Child Neuropsychology: Concepts, Theory, and Practice (pp. 357-374). West Sussex, UK: John Wiley \& Sons.

Butterworth, B., Varma, S., \& Laurillard, D. (2011). Dyscalculia: From brain to education. Science, 332, 1049-1053.

Campbell, K. L., Zimerman, S., Healey, M. K., Lee, M., \& Hasher, L. (2012). Age differences in visual statistical learning. Psychology and Aging, 27, 650-656.

Cantlon, J. F., Platt, M. L., \& Brannon, E. M. (2009). Beyond the number domain. Trends in Cognitive Sciences, 13, 83-91.

Chong, S. C., \& Treisman, A. (2005). Statistical processing: Computing the average size in perceptual groups. Vision Research, 45, 891-900.

Chun, M. M., \& Jiang, Y. (1998). Contextual cueing: Implicit learning and memory of visual context guides spatial attention. Cognitive Psychology, 36, 28-71.

Cohen-Kadosh, R., Cohen Kadosh K., Schumann T., Kaas A., Goebel R., Henik A., \& Sack A. T. (2007). Virtual dyscalculia after TMS to the right parietal lobe: A combined fMRI and neuronavigated TMS study. Current Biology, 17, 689-693. 
Conway, C. M., \& Christiansen, M. H. (2006). Statistical learning within and between modalities: Pitting abstract against stimulus specific representations. Psychological Science, 17, 905-912.

Dehaene, S., Piazza, M., Pinel, P., \& Cohen, L. (2003). Three parietal circuits for number processing. Cognitive Neuropsychology, 20, 487-506.

Emberson, L. L., Conway, C. M., \& Christiansen, M. H. (2011). Timing is everything: Changes in presentation rate have opposite effects on auditory and visual implicit statistical learning. Quarterly Journal of Experimental Psychology, 64, 10211040.

Fiser, J., \& Aslin, R. N. (2001). Unsupervised statistical learning of higher-order spatial structures from visual scenes. Psychological Science, 12, 499-504.

Fiser, J., \& Aslin, R. N. (2002). Statistical learning of higher-order temporal structure from visual shape sequences. Journal of Experimental Psychology: Learning, Memory, and Cognition, 28, 458-467.

Frost, R., Armstrong, B. C., Siegelman, N., \& Christiansen, M. H. (2015). Domain generality versus modality specificity: The paradox of statistical learning. Trends in Cognitive Sciences, 19, 117-125.

Frost, R., Siegelman, N., Narkiss, A., \& Afek, L. (2013). What predicts successful literacy acquisition in a second language? Psychological Science, 24,1243-1252.

Geary, D. C, Bailey, D. H, Littlefield, A., Wood, P., Hoard, M. K., \& Nugent L. (2009). First grade predictors of mathematical learning disability: A latent class trajectory analysis. Cognitive Development, 24, 411-429. 
Gilbert, C. D., Sigman, M., \& Crist, R. E. (2001). The neural basis of perceptual learning. Neuron, 31, 681-697.

Kave, G. (2006). The development of naming and word fluency: Evidence from Hebrewspeaking children between ages 8 and 17. Developmental Neuropsychology, 29, 493-508.

Kirkham, N. Z., Slemmer, J. A., \& Johnson, S. P. (2002). Visual statistical learning in infancy: Evidence for a domain general learning mechanism. Cognition, 83, B35B42.

MATAL. (2007). Diagnostic Battery for the Assessment of Learning Functions. Jerusalem: The Council for Higher Education and the Center for Psychometric Tests.

Musz, E., Weber, M. J., \& Thompson-Schill, S. L. (2015). Visual statistical learning is not reliably modulated by selective attention to isolated events. Attention, Perception, \& Psychophysics, 77, 78-96.

Olshausen, B. A., \& Field, D. J., (1996). Emergence of simple-cell receptive field properties by learning a sparse code for natural images. Nature, 381, 607-609.

Openhaim-Biton, S., \& Breznitz, Z. (2004). 2 minutes calculation test. Unpublished test. University of Haifa, Israel.

Pelli, D. G. (1997). The VideoToolbox software for visual psychophysics: Transforming numbers into movies. Spatial Vision, 10, 437-442.

Piazza, M., Izard, V., Pinel, P., Le Bihan, D., \& Dehaene, S. (2004). Tuning curves for approximate numerosity in the human intraparietal sulcus. Neuron, 44, 547-555. 
Piazza, M., Pinel, P., Le Bihan, D., \& Dehaene, S. (2007). A magnitude code common to numerosities and number symbols in human intraparietal cortex. Neuron, 53, 293-305.

Price, G. R., \& Ansari, D. (2013). Dyscalculia: Characteristics, causes, and treatments. Numeracy, 6, 1-16.

Price, G. R., Holloway, I., Räsänen, P., Vesterinen, M., \& Ansari, D. (2007). Impaired parietal magnitude processing in developmental dyscalculia. Current Biology, 17, 1042-1043.

Raven, J. C. (1960). Guide to the standard progressive matrices. London: H.K. Lewis.

Rotzer, S., Loenneker, T., Kucian, K., Martin, E., Klaver, P., \& Von Aster, M. (2009). Dysfunctional neural network of spatial working memory contributes to developmental dyscalculia. Neuropsychologia, 47, 2859-2865.

Rouder, J. N., Morey, R. D., Speckman, P. L., \& Province, J. M. (2012). Default Bayes factors for ANOVA designs. Journal of Mathematical Psychology, 56, 356-374.

Rubinsten, O. \& Henik, A. (2009). Developmental dyscalculia: Heterogeneity may not mean different mechanisms. Trends in Cognitive Science, 13, 92-99.

Saffran, J. R., Aslin, R. N., \& Newport, E. L. (1996). Statistical learning by 8-month-old infants. Science, 274, 1926-1928.

Shalev, R. S., Auerbach, J., Manor, O., \& Gross-Tsur, V. (2000). Developmental dyscalculia: Prevalence and prognosis. European Child \& Adolescent Psychiatry, 9, S58-S64. 
Shalev, R. S., Manor, O., \& Gross-Tsur, V. (2005). Developmental dyscalculia: A prospective six-year follow-up. Developmental Medicine \& Child Neurology, 47, $121-125$.

Shatil, E. (1997a). One-minute test for words. Unpublished test, University of Haifa, Haifa.

Shatil, E. (1997b). One-minute test for pseudo-words. Unpublished test, University of Haifa, Haifa.

Siegler, R. S., \& Opfer, J. E. (2003). The development of numerical estimation:

Evidence for multiple representations of numerical quantity. Psychological Science, 14, 237-250.

Skagerlund, K., \& Träff, U. (2014). Development of magnitude processing in children with developmental dyscalculia: Space, time, and number. Frontiers in Psychology, 5, 675.

Spencer, M., Kaschak, M. P., Jones, J. L., \& Lonigan, C. J. (2015). Statistical learning is related to early literacy-related skills. Reading and Writing, 28, 467-490.

Szucs, D., Devine, A., Soltesz, F., Nobes, A., \& Gabriel, F. (2013). Developmental dyscalculia is related to visuo-spatial memory and inhibition impairment. Cortex, 49, 2674-2688.

Toro, J. M., \& Trobalón, J. B. (2005). Statistical computations over a speech stream in a rodent. Perception \& Psychophysics, 67, 867-875.

Turk-Browne, N. B., Isola, P. J., Scholl, B. J., \& Treat, T. A. (2008). Multidimensional visual statistical learning. Journal of Experimental Psychology: Learning, Memory, and Cognition, 34, 399-407. 
Turk-Browne, N. B., Jungé, J., \& Scholl, B. J. (2005). The automaticity of visual statistical learning. Journal of Experimental Psychology: General, 134, 552-564.

Turk-Browne, N. B., \& Scholl, B. J. (2009). Flexible visual statistical learning: Transfer across space and time. Journal of Experimental Psychology: Human Perception and Performance, 35, 195-202.

Turk-Browne, N. B., Scholl, B. J., Chun, M. M., \& Johnson, M. K. (2009). Neural evidence of statistical learning: Efficient detection of visual regularities without awareness. Journal of Cognitive Neuroscience, 21, 1934-1945.

Turk-Browne, N. B., Scholl, B. J., Johnson, M. K., \& Chun, M. M. (2010). Implicit perceptual anticipation triggered by statistical learning. Journal of Neuroscience, 30, 1177-1187.

Watanabe, T., Nanez, J. E., \& Sasaki, Y. (2001). Perceptual learning without perception. Nature, 413, 844-848.

Zhao, J., Ngo, N., McKendrick, R., \& Turk-Browne, N. B. (2011). Mutual interference between statistical summary perception and statistical learning. Psychological Science, 22, 1212-1219.

Zhao, J., \& Yu, R. Q. (2016). Statistical regularities reduce perceived numerosity. Cognition, 146, 217-222. 